\title{
Composition, uniqueness and variability of the epiphytic bacterial community of the green alga Ulva australis
}

\author{
Catherine Burke ${ }^{1}$, Torsten Thomas ${ }^{1}$, Matt Lewis ${ }^{2}$, Peter Steinberg ${ }^{3,4}$ and Staffan Kjelleberg ${ }^{1,5}$ \\ ${ }^{1}$ School of Biotechnology and Biomolecular Sciences, Centre for Marine Bio-Innovation, University of \\ New South Wales, Sydney, New South Wales, Australia; ${ }^{2} J$ Craig Venter Institute, Rockville, MD, USA; \\ ${ }^{3}$ School of Biological, Earth and Environmental Sciences, Centre for Marine Bio-Innovation, University \\ of New South Wales, Sydney, New South Wales, Australia; ${ }^{4}$ Sydney Institute of Marine Science, Sydney, \\ New South Wales, Australia and ${ }^{5}$ School of Biological Sciences, Nanyang Technological University, \\ Singapore
}

\begin{abstract}
Green Ulvacean marine macroalgae are distributed worldwide in coastal tidal and subtidal ecosystems. As for many living surfaces in the marine environment, little is known concerning the epiphytic bacterial biofilm communities that inhabit algal surfaces. This study reports on the largest published libraries of near full-length 16S rRNA genes from a marine algal surface (5293 sequences from six samples) allowing for an in-depth assessment of the diversity and phylogenetic profile of the bacterial community on a green Ulvacean alga. Large 16S rRNA gene libraries of surrounding seawater were also used to determine the uniqueness of this bacterial community. The surface of Ulva australis is dominated by sequences of Alphaproteobacteria and the Bacteroidetes, especially within the Rhodobacteriaceae, Sphingomonadaceae, Flavobacteriaceae and Sapropiraceae families. Seawater libraries were also dominated by Alphaproteobacteria and Bacteroidetes sequences, but were shown to be clearly distinct from $U$. australis libraries through the clustering of sequences into operational taxonomic units and Bray-Curtis similarity analysis. Almost no similarity was observed between these two environments at the species level, and only minor similarity was observed at levels of sequence clustering representing clades of bacteria within family and genus taxonomic groups. Variability between libraries of $U$. australis was relatively high, and a consistent sub-population of bacterial species was not detected. The competitive lottery model, originally derived to explain diversity in coral reef fishes, may explain the pattern of colonization of this algal surface.
\end{abstract}

The ISME Journal (2011) 5, 590-600; doi:10.1038/ismej.2010.164; published online 4 November 2010

Subject Category: microbial population and community ecology

Keywords: 16S rRNA gene libraries; epiphytic bacterial community; macroalga; Ulva australis; lottery hypothesis

\section{Introduction}

Marine macroalgae are ecosystem engineers (Jones et al., 1994), playing critical roles in the structuring of coastal communities. Apart from comprising a major component of primary production, macroalgae are habitat-defining organisms by altering sediment matrices and water flow (Alongi, 1998), offering essential space for epibionts (Fraschetti et al., 2006) and providing nurseries and protective environments for many invertebrate species (Wilson et al., 1990; Haywood et al., 1995; Bulleri et al.,

Correspondence: S Kjelleberg, School of Biotechnology and Biomolecular Sciences, Centre for Marine Bio-Innovation, University of New South Wales, Sydney, New South Wales 2052, Australia.

E-mail: s.kjelleberg@unsw.edu.au

Received 31 May 2010; revised 29 July 2010; accepted 18 August 2010; published online 4 November 2010
2002). Indeed, the loss of macroalgal species in coastal ecosystems has been associated with massive decreases in biodiversity (Schiel, 2006; Schiel and Lilley, 2007), highlighting the significance of their function in coastal marine ecosystems.

The surfaces of marine algae also provide a habitat for microbial communities (Bolinches et al., 1988; Jensen et al., 1996; Beleneva and Zhukova, 2006; Wiese et al., 2009), and while negative associations such as disease have been observed (Largo et al., 1999; Vairappan et al., 2001; Wang et al., 2008), the consistent colonization of healthy algae suggests that the normal epiphytic microbiota are interacting with their host in a positive manner. A notable example of such an interaction is the dependence of several green algae on the presence of particular bacteria to develop a normal morphology (Provasoli and Pintner, 1980; Nakanishi et al., 1996; Matsuo et al., 2003; Marshall et al., 2006). Algal-associated 
bacteria have also been shown to induce the release and settlement of algal spores (Joint et al., 2007; Weinberger et al., 2007), to positively influence algal growth and provide essential nutrients (KeshtacherLiebson et al., 1995; Croft et al., 2006), and to induce settlement of sea urchin and other larvae (Johnson and Sutton, 1994; Huggett et al., 2006, 2008). Examples such as these indicate that an epiphytic bacterial community is important to the algal host's normal function and, by extension, the ecology of the habitats in which they exist.

Comprehensive assessments of whole bacterial communities on algal surfaces are relatively rare. The available data, based on $16 S$ rRNA gene sequencing and denaturing gradient gel electrophoresis (DGGE) fingerprinting, suggest that algal associatedcommunities are specific insofar as they differ from the surrounding seawater (Staufenberger et al., 2008) and from other living surfaces (Longford et al., 2007; Lachnit et al., 2009). Given the current lack of data and the importance of algae to coastal ecosystems, it is of great interest to further explore the structure and dynamics of these bacterial communities to understand the ecology of bacterial algal interactions.

The Ulvacean family of marine green macroalgae has a cosmopolitan distribution in coastal areas worldwide (Guiry and Guiry, 2009), and Ulva australis is found commonly in rocky intertidal and sub-tidal areas along the coast of Australia (Womersley, 1984). The surface of $U$. australis hosts a diverse bacterial community (Longford et al., 2007), which is considered to protect the surface of the alga from fouling (Egan et al., 2000). This study reports on an in-depth analysis of the bacterial community of $U$. australis, and a comparison of this bacterial community with that of the surrounding seawater, with the aim of establishing the extent of specificity in community composition between these habitats. Large 16S rRNA gene libraries were constructed from samples of both $U$. australis (six samples and 5293 sequences) and local seawater (10 samples and 10884 sequences) at different time points and locations within the Sydney coastal area, representing to our knowledge the largest study of full-length 16S rRNA gene sequences from an algal surface. The existence of a proposed stable core community of species associated with $U$. australis across space and time (Tujula et al., 2010) is further examined here, along with the distinctness of the community from the surrounding seawater. Finally, we submit that the interpretation of community composition and dynamics of large data sets, as reported in this study, is facilitated by applying a framework of ecological theory.

\section{Methods}

Sample collection, DNA extraction and library construction

$U$. australis thalli (individuals) were collected (wet weight $20 \mathrm{~g}$ per sample) from two different rock pools at Bare Island, La Perouse at low tide in October 2006 (samples UA1 and UA2: $33^{\circ} 59^{\prime} \mathrm{S}$, $151^{\circ} 13^{\prime} \mathrm{E}$ ) and again in February 2007 (samples UA3 and UA4). Thalli were also collected from two different rock pools at Shark Point, Clovelly, in February 2007 (samples UA5 and UA6: $33^{\circ} 91^{\prime}$ S, $151^{\circ} 26^{\prime} \mathrm{E}$ ). Bacterial DNA was extracted from the surface of the algal fronds as described previously (Burke et al., 2009).

Seawater was collected from Sydney Harbour (SW1 and SW2: $33^{\circ} 50^{\prime} \mathrm{S}, 151^{\circ} 15^{\prime} \mathrm{E}$ ) and Botany Bay (SW3 and SW4: $33^{\circ} 59^{\prime} \mathrm{S}, 151^{\circ} 14^{\prime} \mathrm{E}$ ) in January 2005 , and 2 weeks later from Bare Island (SW5 and SW6: $33^{\circ} 59^{\prime} \mathrm{S}, 151^{\circ} 13^{\prime} \mathrm{E}$ ), and Botany Bay (SW7 and SW8). Seawater was collected again from Bare Island (SW9 and SW10) during October 2006 to coincide with the initial sampling of $U$. australis at this site. Two hundred litres of seawater were collected for each sample (100l for SW9 and SW10) from a depth of $2 \mathrm{~m}$, and immediately serially filtered through 20,3 , 0.8 and $0.1 \mu \mathrm{m}$ filters. DNA was extracted from the $0.1 \mu \mathrm{m}$ filter as described previously (Shaw et al., 2008).

16S rRNA gene clone libraries were prepared from DNA extracted from all samples referred to above. 16S rRNA genes were amplified using modified primers 27F (5'-AGRGTTTGATCMTGGCTCAG-3') and 1492R (5'-TACGGYTACCTTGTT AYGACTT-3') using previously described conditions (Shaw et al., 2008). PCR products were cloned into the Zero blunt TOPO PCR cloning kit (Invitrogen, Carlsbad, CA, USA) as per the manufacturer's instructions. Clones were sequenced from both ends, and forward and reverse reads were assembled as in Shaw et al. (2008).

These sequences have been submitted to GenBank under accession numbers: 2266328848-2266331954, 2266325378-2266328847, 2265156107-2265159780, 2265159781-2265163416, 2265142994-2265146681, 2265139436-2265142993, 2271970954-2271974588, 2271978299-2271982008, 2271963732-2271967349, 2271982009-2271985697, 2271974589-2271978298, 2271967350-2271970953, 2271989381-2271993034, 2271985698-2271989380, 21913998 41-2191402367 and 2191386329-2191389151.

Sequences less than $1200 \mathrm{bp}$ in length were removed and the remaining sequences aligned using the SINA web aligner tool (Pruesse et al., 2007). The Bellerophon server (Huber et al., 2004) and the program Mallard (Ashelford et al., 2006) were used to identify putative chimeras. Only sequences identified in both programs as being chimeric were removed from further analysis.

Phylogenetic classification and sequence clustering For a broad comparison of phylogenetic groups between planktonic seawater and $U$. australis libraries, the Ribosomal Database Project (RDP) classifier (Cole et al., 2009) was used with default parameters to classify all sequences. The relative 
abundance of different phyla, family and genus groups within each library was calculated from these results.

A total of 16177 aligned sequences comprising the 16 libraries were used to generate a similarity matrix in ARB (Ludwig et al., 2004). Aligned sequences were clustered by the program Mothur (Schloss et al., 2009) into operational taxonomic units (OTUs) using the furthest neighbour method (that is, any two sequences in one group will have a maximum defined distance). Cutoffs of $0.03,0.05$ and 0.10 difference were used, broadly representing species (0.03), larger clades of bacteria expected to encompass multiple species (0.05) and multiple genera (0.10), within defined taxonomic groups. Mothur was used to calculate the Chao 1 richness estimates at the species (0.03) level of clustering, and to generate Venn diagrams comparing the number of overlapping OTUs between seawater and $U$. australis libraries.

A matrix in the form of raw counts of sequences from each library per OTU was manually constructed for OTUs at the three different taxonomic cutoffs from data extracted from the Mothur analysis. This matrix was analysed using the Primer v5 software package (Clarke and Gorley, 2001) as follows: the data were standardized to account for the different numbers of sequences obtained in each library and used to generate a Bray-Curtis similarity matrix, which was then used to generate dendrograms using the group average.

Assessment of a core community of bacterial species Previous work using molecular fingerprinting techniques has indicated the existence of a group of species that are consistently detected on the surface of different $U$. australis individuals (Tujula et al., 2010), referred to here as a core community. Normalized matrices were examined manually to determine which OTUs occurred consistently across $U$. australis libraries (that is, at least once in each sample). OTUs were classified using the RDP classifier tool, and the level of taxonomic classification chosen included at least $99 \%$ of all sequences for a particular OTU. Representative sequences from each OTU, extracted from Mothur using the get. oturep command, were compared with DGGE bands from a previously proposed stable core community of epiphytic bacteria from the surface of $U$. australis (Tujula et al., 2010) via BLAST (Altschul et al., 1990).

The number of OTUs (at species level, 0.03), which would be expected to be shared across $U$. australis samples by chance, was calculated by randomly assigning each sequence to a sample, mapping each sequence back to the OTU it belongs to, then counting the number of shared OTUs between samples. An average number of shared OTUs were calculated from 50 permutations of this randomization.

\section{Results}

Phylogenetic characterization of planktonic seawater and epiphytic U. australis $16 S$ rRNA gene libraries The 16S rRNA gene libraries of $U$. australis predominantly consisted of sequences from the Proteobacteria (64.0\%) (chiefly the Alphaproteobacteria (54.4\%) and Gammaproteobacteria (8.4\%)), Bacteroidetes (27.6\%) and Planctomycetes (3.4\%); the numbers represent the average percentage of sequences across replicates (Figure 1a). At the level of

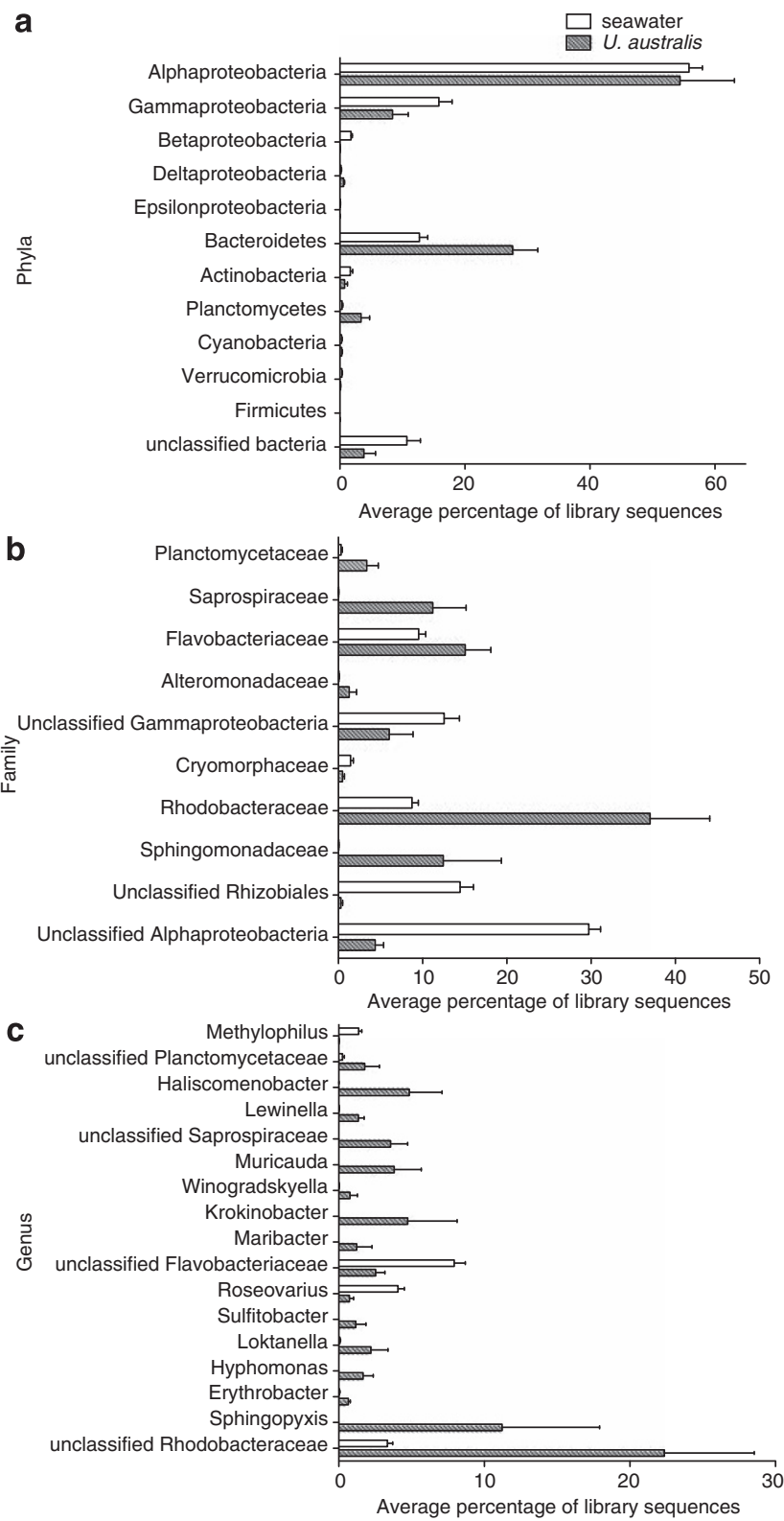

Figure 1 Taxonomic affiliations for sequences within $U$. australis and seawater $16 \mathrm{~S}$ rRNA gene libraries. The average percentage across all $U$. australis and seawater libraries that were classified into particular (a) phyla, (b) family and (c) genus taxonomic groups. Sequences within phyla, family and genus groups, which represented less than $0.01 \%$ of library sequences are not displayed. 
family, sequences fell within the Rhodobacteraceae $(37.0 \%)$ and Sphingomonadaceae (12.5\%) (both Alphaproteobacteria), Flavobacteriaceae (15.0\%) and Saprospiraceae (11.2\%) (both Bacteroidetes) and Planctomycetaceae (3.3\%) (Planctomycetes) as illustrated in Figure 1b; the majority of sequences in the Gammaproteobacteria were unclassified $(6.0 \%)$. At the level of genera, illustrated in Figure 1c, Sphingopyxis (11.2\%), Erythrobacter $(0.7 \%)$, Hyphomonas (1.7\%), Loktanella $(2.2 \%)$, Sulfitobacter $(1.2 \%)$ and a large number of unclassified Rhodobacteriaceae (22.4\%) sequences dominated within the Alphaproteobacteria, and within the Bacteroidetes, genera such as Maribacter $(1.2 \%)$, Muricauda (3.8\%), Krokinobacter (4.7\%), Winogradskyella (0.8\%), Lewinella (1.3\%) and Haliscomenobacter (4.8\%) were prevalent. An average of $3.8 \%$ of sequences in each library were unclassified Bacteria (Figure 1a).

Seawater libraries were dominated by sequences from the Proteobacteria (73.9\%) (Alphaproteobacteria (55.8\%), Gammaproteobacteria (15.8\%), Betaproteobacteria (1.8\%)), Bacteroidetes (12.7\%) and Actinobacteria (1.7\%) phyla, as seen in Figure 1a. At the family level, Alphaproteobacteria sequences consisted of unclassified Rhizobiales $(14.5 \%)$, Rhodobacteraceae $(8.7 \%)$ and unclassified Alphaproteobacteria (29.7\%), whereas the majority of the Bacteroidetes sequences were from the families Flavobacteriaceae (9.5\%) and Cryomorphaceae (1.4\%) (Figure 1b). Gammaproteobacteria sequences were again predominantly unclassified (12.5\%), whereas Betaproteobacteria sequences mostly derived from the Methylophillus genera $(1.4 \%)$. The majority of sequences from seawater libraries were not able to be classified to the genus level with the RDP classifier tool (76.7\%), and an average $10 \%$ of sequences in each library were unclassified Bacteria (Figure 1a).

\section{Clustering of sequences into OTUs and richness} estimation

After removing short and chimeric sequences, the number of sequences in each library ranged from 575 to 1382 (Table 1). Mothur analysis revealed a total of 1061 OTUs across all samples at a cutoff of $0.03 ; 746$ OTUs at 0.05 ; and 353 OTUs at 0.10 difference (Table 1). The number of OTUs at species level (0.03) in individual libraries are listed in Table 1, along with the Chao 1 richness estimate, which ranged from 122 to 642 OTU's per sample.

At species level (0.03), the majority of sequences fell into a relatively small number of OTUs, with $70-80 \%$ of sequences comprising the 30 most abundant OTUs within each library (data not shown). Rarefaction data calculated in Mothur indicated that the number of OTUs detected was steadily increasing with the number of sequences sampled (Figure 2).
Table 1 Number of sequences analysed, OTUs at 0.03 cutoff, and Chao 1 OTU richness estimates for each 16S rRNA gene library

\begin{tabular}{lccc}
\hline Sample & Sequences & OTUs at 0.03 & Chao 1 \\
\hline SW1 & 1148 & 93 & 122 \\
SW2 & 1382 & 107 & 152 \\
SW3 & 1120 & 132 & 161 \\
SW4 & 1179 & 134 & 180 \\
SW5 & 1031 & 177 & 471 \\
SW6 & 972 & 143 & 445 \\
SW7 & 972 & 111 & 167 \\
SW8 & 1279 & 112 & 177 \\
SW9 & 856 & 140 & 642 \\
SW10 & 945 & 184 & 489 \\
UA1 & 790 & 116 & 242 \\
UA2 & 1052 & 135 & 210 \\
UA3 & 957 & 181 & 451 \\
UA4 & 884 & 124 & 225 \\
UA5 & 575 & 117 & 250 \\
UA6 & 1035 & 170 & \\
\end{tabular}

Abbreviation: OTU, operational taxonomic units

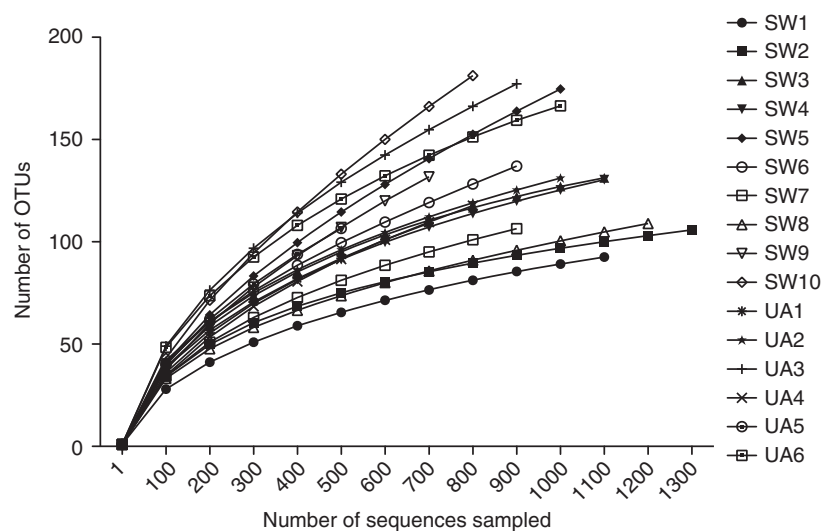

Figure 2 Rarefaction curve. The number of OTUs at 0.03 (species) detected vs the number of sequences sampled in each algal and seawater 16S rRNA gene library.

Differences between epiphytic U. australis and planktonic seawater libraries

Very few OTUs were shared between samples of $U$. australis and seawater (Figure 3). Venn diagrams indicate that $1.88 \%$ of OTUs at 0.03 cutoff and $3.75 \%$ of OTUs at 0.05 cutoff were shared between seawater and $U$. australis libraries. Even at a cutoff of $0.10, U$. australis still contained $87.54 \%$ of OTUs, which were distinct from those in the seawater libraries.

Differences between libraries were also assessed by generating dendrograms from Bray-Curtis similarity matrices, which take into account the abundance of sequences in each OTU (Clarke and Gorley, 2006). The resulting plots for matrices generated from OTUs at (a) 0.03, (b) 0.05 and (c) 0.10 cutoffs are shown in Figure 4. At a 0.03 cutoff or species level, there was almost zero similarity between seawater and $U$. australis libraries, which form distinct clusters. Seawater libraries were approximately $50 \%$ similar to each 
594
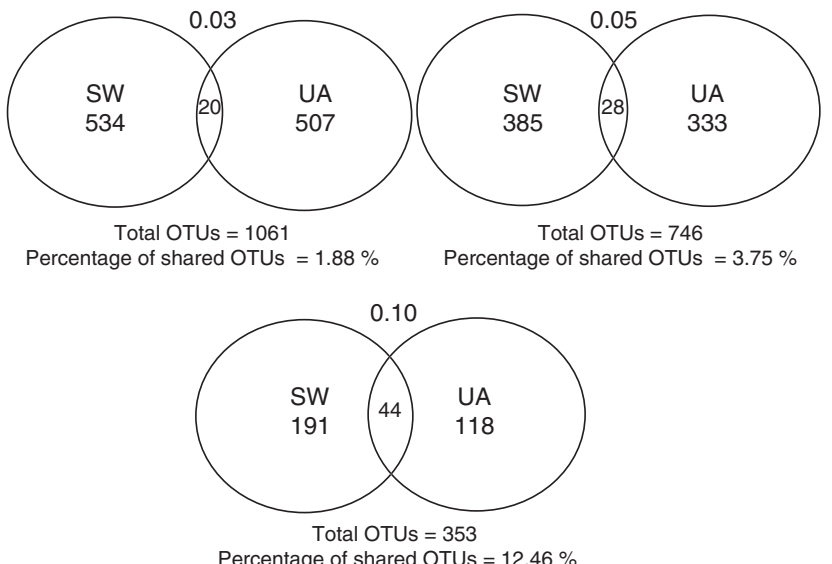

Figure 3 Venn diagrams showing the total number of OTUs and the number of shared OTUs between all $U$. australis (UA) and seawater (SW) libraries at $0.03,0.05$ and 0.10 cutoff.
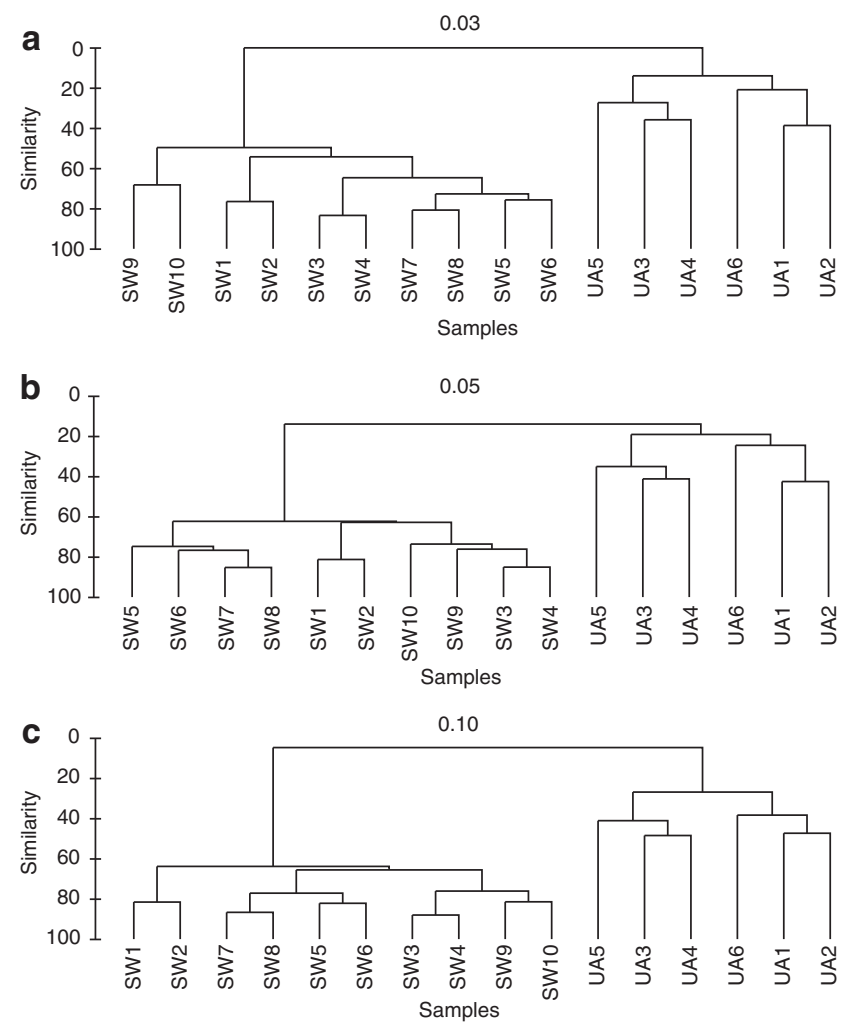

Figure 4 Dendrograms comparing the level of similarity between 10 planktonic and six $U$. australis $16 \mathrm{~S}$ rRNA gene libraries with (a) OTUs at 0.03 difference, (b) OTUs with 0.05 difference and (c) OTUs at 0.10 difference.

other, whereas $U$. australis libraries exhibited a lower degree of similarity with approximately $15 \%$ similarity across all libraries. For dendrograms in Figures $3 \mathrm{~b}$ and $\mathrm{c}$, depicting OTUs clustered at 0.05 and 0.10 cutoffs, the same overall trend was observed. $U$. australis and seawater libraries formed distinct clusters with less than $1 \%$ similarity at 0.05 cutoff and less than $10 \%$ similarity at 0.10 cutoff.
The level of similarity between $U$. australis samples increased to approximately $19 \%$ at 0.05 and $30 \%$ similarity at 0.10 cutoff. Seawater libraries exhibited a higher degree of similarity to each other, with approximately $55 \%$ and $65 \%$ overall similarity at 0.05 and 0.10 cutoffs, respectively.

\section{Is there a core community of epiphytic bacteria} on U. australis?

Species (0.03 cutoff) level OTUs present in all $U$. australis libraries were identified and are listed in Table 2. Remarkably, only six OTUs were consistently present across the six samples, from the 528 total OTUs characterized from U. australis. These included one from the Bacteroidetes (Lewinella sp.) and five from the Alphaproteobacteria (four unclassified Rhodobacteraceae sp. and an Eyrthrobacter sp.). These OTUs accounted for an average of $15.6 \%$ (s.d. \pm 10.96 ) of sequences from each library. The large standard deviation reflects the substantial variation in the number of sequences in each OTU from each $U$. australis library (Table 2). The number of OTUs at species level (0.03), which would be expected by chance alone to be shared across all $U$. australis samples, was 76 (s.d. \pm 3 ), an order of magnitude above what was observed.

In contrast, the seawater libraries contained 21 species level OTUs, which were present in every sample, and these accounted for an average of $66.7 \%$ (s.d \pm 9.6 ) of sequences from each library. A neighbour joining tree of representative OTU and ubiquitous bacterioplankton clade sequences built in ARB (Ludwig et al., 2004) (data not shown) showed that these OTUs cluster with the ubiquitous SAR11 clade (OTUs 1, 4 and 5), the SAR116 clade (OTUs 6, 11, 12, 14, 19 and 20) and the SAR86 clade (OTUs 3, 7, 9 and 16). Other OTUs were classified as belonging to the Flavobacteriaceae family (OTUs 8, 13, 17, 18 and 21) the Deltaproteobacteria class (OTU 15) and the Actinobacteria phylum (OTUs 2 and 10).

Four DGGE sequences proposed to be part of a stable core community of bacteria on $U$. australis in a previous study (Tujula et al., 2010) were compared with sequences from each OTU consistently detected in the $U$. australis libraries. None of the DGGE sequences had high sequence identity (>97\%) to the OTUs present in all libraries reported here.

\section{Discussion}

Living surfaces in the marine environment are hosts to microbial biofilm communities, members of which have been shown to positively affect both the health and normal function of their hosts (Nakanishi et al., 1996; Lee et al., 2001; Lesser et al., 2004; Marshall et al., 2006; Rosenberg et al., 2007). Rather than a random association, increasing evidence suggests that such microbial epiphytic communities are specific to their living hosts 
Table 2 OTUs at species level (0.03), which were found in all $U$. australis libraries

\begin{tabular}{|c|c|c|c|c|c|c|c|}
\hline \multirow[t]{2}{*}{ OTU } & \multirow[t]{2}{*}{ Classification } & \multicolumn{6}{|c|}{ No. of sequences in $O T U$} \\
\hline & & $U A 1$ & $U A 2$ & $U A 3$ & $U A 4$ & UA5 & $U A 6$ \\
\hline 8 & Rhodobacteraceae sp. & $1(0.12 \%)$ & $54(5.13 \%)$ & $231(24.44 \%)$ & $50(5.66 \%)$ & $3(0.52 \%)$ & $54(5.22 \%)$ \\
\hline 12 & Alphaproteobacteria sp. & $26(3.29 \%)$ & $20(1.90 \%)$ & $26(2.75 \%)$ & $3(0.34 \%)$ & $12(2.09 \%)$ & $104(10.05 \%)$ \\
\hline 23 & Rhodobacteraceae sp. & $12(1.52 \%)$ & $14(1.33 \%)$ & $26(2.75 \%)$ & $7(0.79 \%)$ & $17(2.96 \%)$ & $31(3.00 \%)$ \\
\hline 24 & Rhodobacteraceae sp. & $3(0.38 \%)$ & $5(0.48 \%)$ & $12(1.27 \%)$ & $1(0.11 \%)$ & $2(0.53 \%)$ & $80(7.73 \%)$ \\
\hline 45 & Saprospiraceae sp. & $8(1.01 \%)$ & $31(2.95 \%)$ & $5(0.53 \%)$ & $6(0.68 \%)$ & $6(1.04)$ & $3(0.29 \%)$ \\
\hline 97 & Erythrobacter sp. & $8(1.01 \%)$ & $5(0.47 \%)$ & $4(0.42 \%)$ & $4(0.45 \%)$ & $1(0.17 \%)$ & $3(0.29 \%)$ \\
\hline
\end{tabular}

Abbreviations: OTU, operational taxonomic units; RDP, Ribosomal Database Project.

Phylogenetic classification was determined by RDP classifier tool, and the number of sequences per OTU and the percentage of total sequences for each library are also displayed.

(Taylor et al., 2005; Yakimov et al., 2006; Longford et al., 2007; Reis et al., 2009). Such specificity has profound implications for predictions of microbial diversity in the marine environment (Taylor et al., 2004; Longford et al., 2007; Egan et al., 2008), and highlights the importance of investigating such host-associated communities.

Macroalgae are living hosts, performing essential and defining roles in coastal ecosystems (Alongi, 1998; Bulleri et al., 2002; Schiel and Lilley, 2007). However, studies concerning their associated epiphytic bacterial communities are scarce. One example is a recent report on $U$. australis, which was found to have a variable bacterial epiphytic community, but with a stable core community as assessed by consistently occurring DGGE bands (Tujula et al., 2010). However, given the limitations of DGGE (Muyzer et al., 1993), in order to comprehensively address the diversity, variability and uniqueness of the bacterial community on this Ulvacean alga, large 16S rRNA gene libraries of near full-length sequences from multiple samples of $U$. australis across space and time were analysed here, representing the most comprehensive assessment of a macroalgal-associated bacterial community to date. The use of a large number of sequences (5293 from $U$. australis, 10884 from seawater) allowed for the identification of many rare as well as abundant OTUs within both $U$. australis and seawater bacterial communities. This approach is important considering recent evidence that rare phylotypes constitute the majority of diversity in the marine environment (Sogin et al., 2006). The use of near full-length sequences has additionally allowed for a high taxonomic resolution of each library.

Phylogenetic makeup of $\mathrm{U}$. australis and seawater communities

In support of recent studies of surface-associated bacterial communities on sponge and macroalgae (Longford et al., 2007), U. australis and seawater communities were similar at the level of phyla with libraries from both environments dominated by
Proteobacteria- (predominantly Alphaproteobacteria) and Bacteroidetes-affiliated sequences.

$U$. australis libraries were characterized by sequences from the Rhodobacteraceae, Sphingomonadaceae (Alphaproteobacteria), Flavobacteriaceae and Saprospiraceae (Bacteroidetes) families, and included sequences from the Planctomycetaceae (Planctomycetes) and unclassified Gammaproteobacteria. These observations are in broad agreement with the relatively limited data on marine algal-associated bacterial communities. The epiphytic bacterial community of the brown alga Laminaria saccharina was found to be comprised of unclassified Gammaproteobacteria, Flavobacteriaceae and Rhodobacteriaceae sequences (Staufenberger et al., 2008), whereas the red alga Delisea pulchra hosts members of the Rhodobacteraceae, Sphingomonadaceae, Flavobacteriaceae, Planctomycetaceae and unclassified Gammaproteobacteria (Longford et al., 2007). Macrophytes in brackish lakes show a similar community profile (Hempel et al., 2008) and seagrasses have also been found to be associated with a high proportion of Alpha-, Gamma- and Betaproteobacteria, and to a lesser extent with Bacteroidetes (Weidner et al., 2000; Uku et al., 2007). Thus, bacteria within these high-level taxonomic groups may be important associates of algae and marine plants in general.

The phylogenetic affiliations of sequences from the seawater libraries also agree broadly with published studies of coastal prokaryotic planktonic communities (Rappe et al., 2000; Rusch et al., 2007), being dominated by Alphaproteobacteria, Gammaproteobacteria, Bacteroidetes, Actinobacteria and Betaproteobacteria, although Cyanobacteria and Firmicutes are in notably lower abundance here. Many of the seawater sequences were unable to be classified to family or genus level, which is most likely due to these sequences falling into dominant planktonic clades, which are not included in the RDP classification scheme, such as SAR11 (Giovannoni et al., 1990), SAR116 (Mullins et al., 1995) and SAR86 (Fuhrman et al., 1993). This assumption is supported by observations of sequences from the most abundant seawater library OTUs in 
phylogenetic trees, which cluster with published sequences from these clades (data not shown).

U. australis and seawater communities are phylogenetically distinct

Although the seawater and $U$. australis communities have similarities at the level of phyla, the two bacterial communities are strikingly distinct at lower taxonomic levels, as measured by Bray-Curtis similarity and clustering analyses. At the level of species (0.03), communities from the two environments are almost completely dissimilar to one another with close to zero similarity between libraries from the two environments when the abundance of sequences in each OTU are considered (Figure 4a) and less than $2 \%$ of the total OTUs (1061) occurring in both seawater and $U$. australis libraries (Figure 3). This is a surprising result, considering that $U$. australis is for the most part immersed in the surrounding seawater from which these samples were taken. Even more surprising is the level of distinctness observed between the communities at higher levels of sequence clustering, which represent large clades of bacteria within defined taxonomic groups. As the maximum distance between individuals within an OTU increased to 0.05 and 0.10 , these communities remained largely disparate. There was less than $1 \%$ and $11 \%$ similarity across all libraries, respectively (Figures $4 \mathrm{~b}$ and $\mathrm{c}$ ), and the vast majority of OTUs occurred exclusively in one environment or the other (Figure 3). Thus, even at large evolutionary distances, it appears that the bacterial community of $U$. australis is essentially distinct from that of the surrounding seawater, representing a divergent and specific algal-associated community.

Differences in community composition may be due to physical differences between these two environments. Seawater has relatively low nutrient concentrations, whereas living surfaces such as alga exude organic carbon and nutrients (Pregnall, 1983). Bacteria in the seawater must protect themselves from damaging ultraviolet radiation and predation, whereas microbial communities on living surfaces form biofilm structures, shielding them from environmental stress. In addition, living surfaces have particular physical and chemical properties such as morphology (Morrow and Carpenter, 2008) and inhibitory compounds (Steinberg et al., 1997; Kim et al., 2007; Kandhasamy and Arunachalam, 2008), which may act as selective forces on the structure of the bacterial community (Egan et al., 2008; Lam et al., 2008).

The observation that a large number of the OTUs present on the surface of $U$. australis were not detected in the surrounding seawater raises obvious questions as to the origin of these bacteria in the environment before they colonize the surface of $U$. australis. Given richness estimates, these bacteria may be present in the seawater, but below the level of detection, despite the depth of sequencing employed in this work. Alternatively, bacteria may be adapted to a surface-associated lifestyle and attach to small particles or other surfaces in the marine environment, and as such would not have been included in the sampling of the planktonic seawater bacterial community.

\section{Variability within the U. australis community}

On the basis of previous studies, the fact that algal-associated communities were distinct from the surrounding seawater was not completely unexpected. However, the large degree of variability among the $U$. australis libraries was also striking, with the average level of similarity (when the abundance of sequences is considered) less than $20 \%$ at the species level (Figure $4 \mathrm{a}$ ). A recent study of temporal variation on $U$. australis also found that DGGE community profiles from replicate plants shared a minimum $60 \%$ of bands (Tujula et al., 2010). The higher degree of variability observed here is most likely due to the deeper level of sequencing, which has identified many rare OTUs unlikely to be detected by DGGE.

Variability at the species level appears to be an emerging feature of complex host-associated microbial communities in general. For example, the human microbiome project has found high variability at the level of species between human hosts, failing to detect a single species that is present across all human hosts examined (Hamady and Knight, 2009). A more recent study has detected shared species among human hosts, although the abundance of these species varies considerably (Qin et al., 2010). A study of sponges around San Juan Island in the northwestern United States of America showed that bacterial communities could vary considerably for the same species of sponge at different sites (Lee et al., 2009), and a study of the brown alga $L$. saccharina found that while there were strong similarities at higher taxonomic levels, no individual species were consistently found on the algal surface (Staufenberger et al., 2008). The feature of similarities at higher level taxa, such as phyla and class, was also observed in this study and in the human microbiome (Trosvik et al., 2009; Turnbaugh and Gordon, 2009), although the significance of this observation is not clear, as higher level taxonomic groups often encompass a wide variety of functional capabilities (Ettema and Andersson, 2009). The congruence between these human studies and marine systems may reveal an emerging principle of host-associated, complex microbial communities.

\section{Lack of a core community of bacterial species on} U. australis

In a previous study of the $U$. australis epiphytic bacterial community, it was proposed that while the 
community is variable, there is a core community of species that are found consistently on the algal surface (Tujula et al., 2010). A small number of OTUs (six) were detected in all $U$. australis libraries, although the abundance of sequences within each OTU varied widely (Table 2). Although they correspond to the same broad groups (phyla and families) identified as important in our previous study, none of the consistently detected OTUs from this study had $97 \%$ similarity or more to DGGE sequences, which previously appeared to comprise the core community of bacterial species. This discrepancy may be due to the different techniques employed; in the previous study, different primers for a fragment (around $560 \mathrm{bp}$ ) of the 16S rRNA gene were used, as compared to almost full-length sequences here (at least $1200 \mathrm{bp}$ ). In addition, different DNA extraction methods were employed in the two studies, although these have been shown to give no significant difference in community composition when applied to $U$. australis (Burke et al., 2009). The most obvious difference between the previous study and this one is the amount of data that was generated and analysed, highlighting the increased resolving power that comes with deeper sequencing of environmental microbial communities (Sogin et al., 2006). On the basis of the data presented here, there does not appear to be a consistent core community of bacterial species colonizing the surface of $U$. australis, suggesting that a large number of bacterial species are able to colonize this algal surface.

Functional redundancy and the lottery hypothesis The high variability of community membership between different samples of $U$. australis suggests that functional redundancy exists within this community. This conclusion is consistent with the redundancy hypothesis (Naeem, 1998), which assumes that more than one species is capable of performing a specific role within an ecosystem, conferring ecosystem functions a degree of resilience to disturbance. Although originally proposed for communities of macroorganisms, this hypothesis has been tested and supported for microbial communities both in laboratory microcosms (Bell et al., 2005; Leflaive et al., 2008) and in situ soil communities (Yin et al., 2000; Persiani et al., 2008). Functional redundancy has also been shown in algal-associated bacterial communities, in which bacteria from a range of taxa within the community were shown to be able to induce normal thallus morphology on the green alga Ulva linza (Marshall et al., 2006). Although functional redundancy may account for the variability observed within the $U$. australis bacterial community, on its own, it does not account for the striking differences observed between this algal-associated community and the planktonic community of the surrounding seawater. These two communities are clearly distinct, even when considering OTUs with large phylogenetic distances (0.05 and 0.10). This suggests that there are selective mechanisms in place, which determine the assemblage of species that exist in one environment or the other. How can these observations of both selectiveness and variability be reconciled?

The lottery hypothesis (Sale, 1976), developed to explain the coexistence of reef fish species that occupy the same niche, fits the observations described here. This hypothesis asserts that species with similar trophic abilities will occupy space within an ecosystem based on stochastic recruitment. That is, within a group of species with similar ecologies, whoever gets there first wins the lottery for space (Kelley, 1989; Munday, 2004). Thus, groups of similar species will only occupy a new niche if it is empty or vacated by another species.

The lottery hypothesis bears resemblance to Hubbell's neutral theory, a model of community assembly that departs from the traditional nichebased view by assuming that trophically similar species are ecologically equivalent (Hubbell, 2001), and that the composition of communities at a local scale is influenced only by random immigration, birth and death events (Woodcock et al., 2007). Neutral models have been shown to accurately predict species distribution curves for both tropical tree communities (Hubbell, 2005) and those of coral reefs (Volkov et al., 2007). Neutral models of community assembly have also been applied to bacterial communities, with similarly accurate predictive power with respect to species abundance distributions (Sloan et al., 2006, 2007; Woodcock et al., 2007). Although the existence of niche differences between different species is not disputed by these authors, they propose a larger role for stochastic processes in the assembly of ecological communities than has previously been argued.

Neutral models and the lottery hypothesis both work on the assumption of ecological equivalence, and the neutral model assumes this broadly. In contrast, the lottery hypothesis makes this assumption for defined groups of species sharing a particular niche (for example, Ulva vs seawater) or functional aspect. In the case of $U$. australis, this model would assert that there is a guild of bacteria that all possess the necessary metabolic abilities to colonize the various niches on the algal surface. By definition within this guild, there is functional redundancy and whichever species from the guild happen to encounter and occupy the surface first are those that will colonize it. This would account for the variability observed between $U$. australis samples. Species from the $U$. australis guild may not be adapted to survive and flourish planktonically, which could explain why bacterial species found on the surface of $U$. australis are rarely found in the surrounding seawater. One implication of the lottery hypothesis for this system is that the total species pool or guild that is capable of colonizing the $U$. australis surface is very large, 
much larger than that observed here. The number of observed species shared among all algal samples was six, an order of magnitude lower than that expected by chance (76) from randomly assigning observed sequences in this data set to the six Ulva samples. Given that the lottery hypothesis was originally conceived for communities in which guild size was some dozens of species of fish, it will be interesting to see if this hypothesis can also apply to guilds that potentially contain thousands of species of bacteria. Furthermore, it would be of great interest to know whether the 'guild' of bacteria observed here is specific to $U$. australis, to algae, or is simply indicative of a surface-associated lifestyle, and this can only be ascertained by comparing the communities of other algal, living and inanimate surfaces, and a variety of non-surface-associated bacterial communities.

\section{Acknowledgements}

This work was funded by the Australian Research Council; the Gordon and Betty Moore Foundation; the US Department of Energy (DOE), Office of Science, Office of Biological and Environmental Research (DE-FG0202ER63453); and the J Craig Venter Institute, USA.

\section{References}

Alongi DM. (1998). Coastal Ecosystem Processes. CC Press: London.

Altschul SF, Gish W, Miller W, Myers EW, Lipman DJ. (1990). Basic local alignment search tool. J Mol Biol 215: 403-410.

Ashelford KE, Chuzhanova NA, Fry JC, Jones AJ, Weightman AJ. (2006). New screening software shows that most recent large $16 \mathrm{~S}$ rRNA gene clone libraries contain chimeras. Appl Environ Microbiol 72: 5734-5741.

Beleneva IA, Zhukova NV. (2006). Bacterial communities of some brown and red algae from Peter the Great Bay, the Sea of Japan. Microbiology 75: 348-357.

Bell T, Newman JA, Silverman BW, Turner SL, Lilley AK. (2005). The contribution of species richness and composition to bacterial services. Nature 436: 1157-1160.

Bolinches J, Lemos ML, Barja JL. (1988). Populationdynamics of heterotrophic bacterial communities associated with Fucus vesiculosus and Ulva rigida in an estuary. Microb Ecol 15: 345-357.

Bulleri F, Benedetti-Cecchi L, Acunto S, Cinelli F, Hawkins SJ. (2002). The influence of canopy algae on vertical patterns of distribution of low-shore assemblages on rocky coasts in the northwest Mediterranean. J Exp Mar Biol Ecol 267: 89-106.

Burke C, Kjelleberg S, Thomas T. (2009). Selective extraction of bacterial DNA from the surfaces of macroalgae. Appl Environ Microbiol 75: 252-256.

Clarke KR, Gorley RN. (2001). PRIMER v5: User Manual/ Tutorial. PRIMER-E: Plymoth.

Clarke KR, Gorley RN. (2006). PRIMER v6: User Manual/ Tutorial. PRIMER-E: Plymoth.
Cole JR, Wang Q, Cardenas E, Fish J, Chai B, Farris RJ et al. (2009). The ribosomal database project: improved alignments and new tools for rRNA analysis. Nucleic Acids Res 37: D141-D145.

Croft MT, Warren MJ, Smith AG. (2006). Algae need their vitamins. Eukaryot Cell 5: 1175-1183.

Egan S, Thomas T, Holmstrom C, Kjelleberg S. (2000). Phylogenetic relationship and antifouling activity of bacterial epiphytes from the marine alga Ulva lactuca. Environ Microbiol 2: 343-347.

Egan S, Thomas T, Kjelleberg S. (2008). Unlocking the diversity and biotechnological potential of marine surface associated microbial communities. Curr Opin Microbiol 11: 219-225.

Ettema TJG, Andersson SGE. (2009). The alphaproteobacteria: the Darwin finches of the bacterial world. Biol Lett 5: 429-432.

Fraschetti S, Terlizzi A, Bevilacqua S, Boero F. (2006). The distribution of hydroids (Cnidaria, Hydrozoa) from micro- to macro-scale: spatial patterns on habitatforming algae. J Exp Mar Biol Ecol 339: 148-158.

Fuhrman JA, McCallum K, Davis AA. (1993). Phylogenetic diversity of subsurface marine microbial communities from the Atlantic and Pacific oceans. Appl Environ Microbiol 59: 1294-1302.

Giovannoni SJ, Britschgi TB, Moyer CL, Field KG. (1990). Genetic diversity in Sargasso Sea bacterioplankton. Nature 345: 60-63.

Guiry MD, Guiry GM. (2009) National University of Ireland: Galway.

Hamady M, Knight R. (2009). Microbial community profiling for human microbiome projects: tools, techniques, and challenges. Genome Res 19: 1141-1152.

Haywood MDE, Vance DJ, Loneragan NR. (1995). Seagrass and algal beds as nursery habitats for tiger prawns (Penaeus semisulcatus and P. esculentus) in a tropical Australian estuary. Mar Biol 122: 213-223.

Hempel M, Blume M, Blindow I, Gross EM. (2008). Epiphytic bacterial community composition on two common submerged macrophytes in brackish water and freshwater. BMC Microbiol 8: 10.

Hubbell SP. (2001). The Unified Neutral Theory of Biodiversity and Biogeography. Princeton Univeristy Press: Princeton, NJ.

Hubbell SP. (2005). Neutral theory in community ecology and the hypothesis of functional equivalence. Funct Ecol 19: 166-172.

Huber T, Faulkner G, Hugenholtz P. (2004). Bellerophon: a program to detect chimeric sequences in multiple sequence alignments. Bioinformatics 20: 2317-2319.

Huggett MJ, Crocetti GR, Kjelleberg S, Steinberg PD. (2008). Recruitment of the sea urchin Heliocidaris erythrogramma and the distribution and abundance of inducing bacteria in the field. Aquat Microb Ecol 53: 161-171.

Huggett MJ, Williamson JE, de Nys R, Kjelleberg S, Steinberg PD. (2006). Larval settlement of the common Australian sea urchin Heliocidaris erythrogramma in response to bacteria from the surface of coralline algae. Oecologia 149: 604-619.

Jensen PR, Kauffman CA, Fenical W. (1996). High recovery of culturable bacteria from the surfaces of marine algae. Mar Biol 126: 1-7.

Johnson CR, Sutton DC. (1994). Bacteria on the surface of crustose coralline algae induce metamorphosis of the crown-of-thorns starfish Acanthaster planci. Mar Biol 120: $305-310$. 
Joint I, Tait K, Wheeler G. (2007). Cross-kingdom signalling: exploitation of bacterial quorum sensing molecules by the green seaweed Ulva. Philos Trans Roy Soc B 362: 1223-1233.

Jones CG, Lawton JH, Shachak M. (1994). Organisms as ecosystem engineers. Oikos 69: 373-386.

Kandhasamy M, Arunachalam KD. (2008). Evaluation of in vitro antibacterial property of seaweeds of southeast coast of India. Afr J Biotechnol 7: 1958-1961.

Kelley SE. (1989). Experimental studies of the evolutionary significance of sexual reproduction 5. A field-test of the sib-competition lottery hypothesis. Evolution 43: 1054-1065.

Keshtacher-Liebson E, Hadar Y, Chen Y. (1995). Oligotrophic bacteria enhance algal growth under iron-deficient conditions. Appl Environ Microbiol 61: 2439-2441.

Kim IH, Lee DG, Lee SH, Ha JM, Ha BJ, Kim SK et al. (2007). Antibacterial activity of Ulva lactuca against methicillin-resistant Staphylococcus aureus (MRSA). Biotechnol Bioproc Eng 12: 579-582.

Lachnit T, Blumel M, Imhoff JF, Wahl M. (2009). Specific epibacterial communities on macroalgae: phylogeny matters more than habitat. Aquat Biol 5: 181-186.

Lam C, Stang A, Harder T. (2008). Planktonic bacteria and fungi are selectively eliminated by exposure to marine macroalgae in close proximity. FEMS Microbiol Ecol 63: $283-291$.

Largo DB, Fukami K, Nishijima T. (1999). Time-dependent attachment mechanism of bacterial pathogen during ice-ice infection in Kappaphycus alvarezii (Gigartinales, Rhodophyta). J Appl Phycol 11: 129-136.

Lee OO, Wong YH, Qian PY. (2009). Inter- and intraspecific variations of bacterial communities associated with marine sponges from San Juan Island, Washington. Appl Environ Microbiol 75: 3513-3521.

Lee YK, Lee JH, Lee HK. (2001). Microbial symbiosis in marine sponges. J Microbiol 39: 254-264.

Leflaive J, Danger M, Lacroix G, Lyautey E, Oumarou C, Ten-Hage L. (2008). Nutrient effects on the genetic and functional diversity of aquatic bacterial communities. FEMS Microbiol Ecol 66: 379-390.

Lesser MP, Mazel CH, Gorbunov MY, Falkowski PG. (2004). Discovery of symbiotic nitrogen-fixing cyanobacteria in corals. Science 305: 997-1000.

Longford SR, Tujula NA, Crocetti GR, Holmes AJ, Holmstrom C, Kjelleberg S et al. (2007). Comparisons of diversity of bacterial communities associated with three sessile marine eukaryotes. Aquat Microb Ecol 48: 217-229.

Ludwig W, Strunk O, Westram R, Richter L, Meier H, Yadhukumar et al. (2004). ARB: a software environment for sequence data. Nucleic Acids Res 32: 1363-1371.

Marshall K, Joint I, Callow ME, Callow JA. (2006). Effect of marine bacterial isolates on the growth and morphology of axenic plantlets of the green alga Ulva linza. Microb Ecol 52: 302-310.

Matsuo Y, Suzuki M, Kasai H, Shizuri Y, Harayama S. (2003). Isolation and phylogenetic characterization of bacteria capable of inducing differentiation in the green alga Monostroma oxyspermum. Environ Microbiol 5: 25-35.

Morrow KM, Carpenter RC. (2008). Macroalgal morphology mediates particle capture by the corallimorpharian Corynactis californica. Mar Biol 155: 273-280.
Mullins TD, Britschgi TB, Krest RL, Giovannoni SJ. (1995). Genetic comparisons reveal the same unknown bacterial lineages in Atlantic and Pacific bacterioplankton communities. Limnol Oceanogr 40: 148-158.

Munday PL. (2004). Competitive coexistence of coraldwelling fishes: the lottery hypothesis revisited. Ecology 85: 623-628.

Muyzer G, Dewaal EC, Uitterlinden AG. (1993). Profiling of complex microbial populations by denaturing gradient gel electrophoresis analysis of polymerase chain reaction amplified genes coding for $16 \mathrm{~S}$ ribosomal RNA. Appl Environ Microbiol 59: 695-700.

Naeem S. (1998). Species redundancy and ecosystem reliability. Conserv Biol 12: 39-45.

Nakanishi K, Nishijima M, Nishimura M, Kuwano K, Saga N. (1996). Bacteria that induce morphogenesis in Ulva pertusa (Chlorophyta) grown under axenic conditions. J Phycol 32: 479-482.

Persiani AM, Maggi O, Montalvo J, Casado MA, Pineda FD. (2008). Mediterranean grassland soil fungi: patterns of biodiversity, functional redundancy and soil carbon storage. Plant Biosyst 142: 111-119.

Pregnall AM. (1983). Release of dissolved organic-carbon from the estuarine intertidal macroalga Enteromorpha prolifera. Mar Biol 73: 37-42.

Provasoli L, Pintner IJ. (1980). Bacteria induced polymorphism in an axenic laboratory strain of Ulva lactuca (Chlorophyceae). J Phycol 16: 196-201.

Pruesse E, Quast C, Knittel K, Fuchs BM, Ludwig WG, Peplies J et al. (2007). SILVA: a comprehensive online resource for quality checked and aligned ribosomal RNA sequence data compatible with ARB. Nucleic Acids Res 35: 7188-7196.

Qin JJ, Li RQ, Raes J, Arumugam M, Burgdorf KS, Manichanh C et al. (2010). A human gut microbial gene catalogue established by metagenomic sequencing. Nature 464: 59-U70.

Rappe MS, Vergin K, Giovannoni SJ. (2000). Phylogenetic comparisons of a coastal bacterioplankton community with its counterparts in open ocean and freshwater systems. FEMS Microbiol Ecol 33: 219-232.

Reis AMM, Araujo SD, Moura RL, Francini RB, Pappas G, Coelho AMA et al. (2009). Bacterial diversity associated with the Brazilian endemic reef coral Mussismilia braziliensis. J Appl Microbiol 106: 1378-1387.

Rosenberg E, Koren O, Reshef L, Efrony R, ZilberRosenberg I. (2007). The role of microorganisms in coral health, disease and evolution. Nat Rev Microbiol 5: 355-362.

Rusch DB, Halpern AL, Sutton G, Heidelberg KB, Williamson S, Yooseph S et al. (2007). The Sorcerer II global ocean sampling expedition: Northwest Atlantic through Eastern Tropical Pacific. PLoS Biol 5: $398-431$.

Sale PF. (1976). Reef fish lottery. Nat History 85: 60-65.

Schiel DR. (2006). Rivets or bolts? when single species count in the function of temperate rocky reef communities. J Exp Mar Biol Ecol 338: 233-252.

Schiel DR, Lilley SA. (2007). Gradients of disturbance to an algal canopy and the modification of an intertidal community. Mar Ecol Progr Ser 339: 1-11.

Schloss PD, Westcott SL, Ryabin T, Hall JR, Hartmann M, Hollister EB et al. (2009). Introducing mothur: open-source, platform-independent, communitysupported software for describing and comparing microbial communities. Appl Environ Microbiol 75: 7537-7541. 
Shaw AK, Halpern AL, Beeson K, Tran B, Venter JC, Martiny JBH. (2008). It's all relative: ranking the diversity of aquatic bacterial communities. Environ Microbiol 10: 2200-2210.

Sloan WT, Lunn M, Woodcock S, Head IM, Nee S, Curtis TP. (2006). Quantifying the roles of immigration and chance in shaping prokaryote community structure. Environ Microbiol 8: 732-740.

Sloan WT, Woodcock S, Lunn M, Head IM, Curtis TP. (2007). Modeling taxa-abundance distributions in microbial communities using environmental sequence data. Microb Ecol 53: 443-455.

Sogin ML, Morrison HG, Huber JA, Mark Welch D, Huse SM, Neal PR et al. (2006). Microbial diversity in the deep sea and the underexplored 'rare biosphere'. Proc Natl Acad Sci USA 103: 12115-12120.

Staufenberger T, Thiel V, Wiese J, Imhoff JF. (2008). Phylogenetic analysis of bacteria associated with Laminaria saccharina. FEMS Microbiol Ecol 64: 65-77.

Steinberg PD, Schneider R, Kjelleberg S. (1997). Chemical defenses of seaweeds against microbial colonization. Biodegradation 8: 211-220.

Taylor MW, Schupp PJ, Dahllof I, Kjelleberg S, Steinberg PD. (2004). Host specificity in marine spongeassociated bacteria, and potential implications for marine microbial diversity. Environ Microbiol 6: 121-130.

Taylor MW, Schupp PJ, de Nys R, Kjelleberg S, Steinberg PD. (2005). Biogeography of bacteria associated with the marine sponge Cymbastela concentrica. Environ Microbiol 7: 419-433.

Trosvik P, Stenseth NC, Rudi K. (2009). Convergent temporal dynamics of the human infant gut microbiota. ISME J 4: 151-158.

Tujula NA, Crocetti GR, Burke C, Thomas T, Holmstrom C, Kjelleberg S. (2010). Variability and abundance of the epiphytic bacterial community associated with a green marine Ulvacean alga. ISME J 4: 301-311.

Turnbaugh PJ, Gordon JI. (2009). The core gut microbiome, energy balance and obesity. J Physiol Lond 587: 4153-4158.

Uku J, Bjork M, Bergman B, Diez B. (2007). Characterization and comparison of prokaryotic epiphytes associated with three east african seagrasses. J Phycol 43: 768-779.
Vairappan CS, Suzuki M, Motomura T, Ichimura T. (2001). Pathogenic bacteria associated with lesions and thallus bleaching symptoms in the Japanese kelp Laminaria religiosa Miyabe (Laminariales, Phaeophyceae). Hydrobiologia 445: 183-191.

Volkov I, Banavar JR, Hubbell SP, Maritan A. (2007). Patterns of relative species abundance in rainforests and coral reefs. Nature 450: 45-49.

Wang G, Shuai L, Li Y, Lin W, Zhao XW, Duan DL. (2008). Phylogenetic analysis of epiphytic marine bacteria on Hole-Rotten diseased sporophytes of Laminaria japonica. J Appl Phycol 20: 403-409.

Weidner S, Arnold W, Stackebrandt E, Puhler A. (2000). Phylogenetic analysis of bacterial communities associated with leaves of the seagrass Halophila stipulacea by a culture-independent small-subunit rRNA gene approach. Microb Ecol 39: 22-31.

Weinberger F, Beltran J, Correa JA, Lion U, Pohnert G, Kumar N et al. (2007). Spore release in Acrochaetium sp. (Rhodophyta) is bacterially controlled. J Phycol 43: 235-241.

Wiese J, Thiel V, Nagel K, Staufenberger T, Imhoff JF. (2009). Diversity of antibiotic-active bacteria associated with the brown alga Laminaria saccharina from the Baltic Sea. Mar Biotechnol 11: 287-300.

Wilson KA, Able KW, Heck KL. (1990). Predation rates on juvenile blue crabs in estuarine nursery habitatsevidence for the importance of macroalgae (Ulva lactuca). Mar Ecol Progr Ser 58: 243-251.

Womersley H. (1984). The Marine Benthic Flora of Southern Australia, vol 1. Australian Biological Resources Study: Adelaide.

Woodcock S, van der Gast CJ, Bell T, Lunn M, Curtis TP, Head IM et al. (2007). Neutral assembly of bacterial communities. FEMS Microbiol Ecol 62: 171-180.

Yakimov MM, Cappello S, Crisafi E, Tursi A, Savini A, Corselli C et al. (2006). Phylogenetic survey of metabolically active microbial communities associated with the deep-sea coral Lophelia pertusa from the Apulian plateau, Central Mediterranean Sea. DSRI 53: 62-75.

Yin B, Crowley D, Sparovek G, De Melo WJ, Borneman J. (2000). Bacterial functional redundancy along a soil reclamation gradient. Appl Environ Microbiol 66: 4361-4365. 